\title{
Artificial association of memory events by optogenetic stimulation of hippocampal CA3 cell ensembles
}

\author{
Naoya Oishi ${ }^{1,2}$, Masanori Nomoto ${ }^{1,2}$, Noriaki Ohkawa ${ }^{1,2,3}$, Yoshito Saitoh ${ }^{1,2}$, Yoshitake Sano ${ }^{1,2,7}$, Shuhei Tsujimura ${ }^{1,2}$,
} Hirofumi Nishizono ${ }^{2,4}$, Mina Matsuo ${ }^{4}$, Shin-ichi Muramatsu ${ }^{5,6}$ and Kaoru Inokuchi ${ }^{1,2^{*}}$

\begin{abstract}
Previous gain-of-function studies using an optogenetic technique showed that manipulation of the hippocampal dentate gyrus or CA1 cell ensembles is important for memory reactivation and to generate synthetic or false memory. However, gain-of-function study manipulating CA3 cell ensembles has not been reported. The CA3 area of the hippocampus comprises a recurrent excitatory circuit, which is thought to be important for the generation of associations among the stored information within one brain region. We investigated whether the coincident firing of cell ensembles in one brain region, hippocampal CA3, associates distinct events. CA3 cell ensembles responding to context exploration and during contextual fear conditioning were labeled with channelrhodopsin2 (ChR2)-mCherry. The synchronous activation of these ensembles induced freezing behavior in mice in a neutral context, in which a foot shock had never been delivered. The recall of this artificial associative fear memory was context specific. In vivo electrophysiological recordings showed that 20-Hz optical stimulation of ChR2-mCherryexpressing CA3 neurons, which is the same stimulation protocol used in behavioral experiment, induced long-term potentiation at CA3-CA3 synapses. Altogether, these results demonstrate that the synchronous activation of ensembles in one brain region, CA3 of the hippocampus, is sufficient for the association of distinct events. The results of our electrophysiology potentially suggest that this artificial association of memory events might be induced by the strengthening of synaptic efficacy between CA3 ensembles via recurrent circuit.
\end{abstract}

Keywords: Hippocampus, CA3, Recurrent circuit, Artificial association, Synaptic plasticity, Long-term potentiation (LTP), Optogenetics

\section{Introduction}

Subpopulation of neurons that were activated during learning, is reactivated during retrieval [1-6] and that activation of the specific neuronal ensemble is required and sufficient to retrieve that memory [7-10]. These findings indicate that memories are stored in cell ensemble activated during learning.

Acquisition of a new memory is susceptible to modification by the simultaneous and artificial activation of a specific neural ensemble corresponding to that pre-stored

\footnotetext{
* Correspondence: inokuchi@med.u-toyama.ac.jp

'Department of Biochemistry, Graduate School of Medicine and

Pharmaceutical Sciences, University of Toyama, Toyama 930-0194, Japan

${ }^{2}$ Core Research for Evolutional Science and Technology (CREST), Japan

Science and Technology Agency (JST), University of Toyama, Toyama

930-0194, Japan

Full list of author information is available at the end of the article
}

memory, generating synthetic or false memories $[4,11]$. Retrieval of two independent memories by natural cue or optogenetic stimulation associates distinct events $[12,13]$.

Coincident activation of neurons results in a strengthening in synaptic efficacy such as long-term potentiation (LTP) $[14,15]$. LTP at appropriate synapses are both necessary and sufficient for information storage $[16,17]$, and it also contributes to associative learning or memory update [18-20].

Previous gain-of-function studies using an optogenetic technique showed that manipulation of the hippocampal dentate gyrus (DG) [4, 8, 21-24] or CA1 [12] cell ensembles is important for memory reactivation and to generate synthetic or false memory by linking between stored information and sensory input by artificial activation of cell

(c) The Author(s). 2019 Open Access This article is distributed under the terms of the Creative Commons Attribution 4.0 International License (http://creativecommons.org/licenses/by/4.0/), which permits unrestricted use, distribution, and 
ensembles. However, gain-of-function study manipulating hippocampal CA3 cell ensembles has not been reported.

Pyramidal cells in the CA3 region of the hippocampus make synapses with each other via recurrent collaterals $[25,26]$. This recurrent excitatory circuit has a key role in retrieving whole pattern from degraded cue, a process called pattern completion [27-29]. The hippocampal CA3 is reported to have an important role in the associative learning [30,31], and this recurrent network is thought to form the associative memory within one brain region [30]. However, there is no experimental evidence demonstrating that CA3 region is important for the incorporation of previously stored information within one brain region to generate associative memories.

We hypothesized that the coincident firing of cell ensembles of the hippocampal CA3, which have recurrent network within one brain region, integrates distinct events. Also, we tested whether the synchronous activation of CA3 induces LTP in CA3-CA3 synapses. Here we showed that the synchronous activation of ensembles in CA3 associates distinct events. Also, in vivo electrophysiological recording showed that $20-\mathrm{Hz}$ optical stimulation of ChR2-mCherry expressing CA3 neurons, which is the same stimulation protocol used in behavioral experiment, induces LTP in CA3-CA3 synapses. This results of electrophysiology potentially suggest that the artificial association of memory events might be induced by the strengthening of synaptic efficacy between CA3 ensembles via recurrent circuit.

\section{Methods}

\section{Animals and genotyping}

The c-fos::tetracycline transactivator (tTA) mice were purchased from the Mutant Mouse Regional Resource Center (stock no. 031756-MU). The KA1::Cre mice were purchased from Jackson Laboratory (Jackson Laboratory stock no: 006474, G32-4 Cre). Floxed-NR1 mice (Jackson Laboratory stock no: 005246) were donated by Drs. S. Tonegawa (RIKEN-Massachusetts Institute of Technology) and S. Itohara (RIKEN Brain Science Institute).

The c-fos::tTA/KA1::Cre double transgenic mice for behavioral analyses were generated via in vitro fertilization with eggs from C57BL/6 J mice and embryo transfer techniques [12]. The CA3 pyramidal cell-restricted $N$-methylD-aspartate (NMDA) receptor knock-out (CA3-NR1 KO) mice were generated from KA1::Cre mice and homozygous floxed-NR1 mice via in vitro fertilization.

The c-fos::tTA mice were backcrossed with C57BL/6 J at least 10 times after we purchased from the Mutant Mouse Regional Resource Center. The KA1::Cre mice was mixed C57BL/6 J and C57BL/6 N genetic background when we purchased from Jackson Laboratory. The results of A 32 SNP (single nucleotide polymorphism) panel analysis, which conducted by Jackson Laboratory showed that, at least 3 of 5 markers that distinguish C57BL/6 J from
C57BL/6 $\mathrm{N}$ were found to be C57BL/6 J type [32]. After we obtained the KA1::Cre mice, the mice were backcrossed with $\mathrm{C} 57 \mathrm{BL} / 6 \mathrm{~J}$ at least 5 times to be $\mathrm{C} 57 \mathrm{BL} / 6 \mathrm{~J}$ background. The fNR1 mice were backcrossed with C57BL/6 J more than 25 times. Therefore, all experimental mice were C57BL/6 J background. Male mice were used for all the experiment.

The mice were maintained on a 12-h light-dark cycle (lights on $8: 00 \mathrm{am}$ ) at $24 \pm 3{ }^{\circ} \mathrm{C}$ and $55 \pm 5 \%$ humidity with food and water ad libitum and were housed with littermates until the surgeries. All procedures involving the use of animals complied with the guidelines of the National Institutes of Health and were approved by the Animal Care and Use Committee of the University of Toyama.

c-fos::tTA mice were genotyped via polymerase chain reaction (PCR) with genomic DNA isolated from the tails of the pups as described previously [33]. To detect the Cre recombinase transgene in KA1::Cre mice, 5 -ACCTGA TGGACATGTTCAGGGATCG-3' and 5'-TCCGGTTAT TCAACTTGCACCATGC-3' primers were used with PCR conditions of $94^{\circ} \mathrm{C}$ for $2 \mathrm{~min}, 35$ cycles of $94^{\circ} \mathrm{C}$ for $30 \mathrm{~s}, 55^{\circ} \mathrm{C}$ for $15 \mathrm{~s}$, and $72{ }^{\circ} \mathrm{C}$ for $20 \mathrm{~s}$, followed by $72^{\circ} \mathrm{C}$ for $7 \mathrm{~min}$. For floxed-NR1 mice, the primers $5^{\prime}$-GCTT GGGTGGAGAGGCTATTC-3' and 5'-CAAGGTGAG ATGACAGGAGATC-3' to detect the neomycin resistance cassette and 5' -TGTGCTGGGTGTGAGGGTTG-3' and $5^{\prime}$-GTGAGCTGCACTTCCAGAAG-3' to detect the NR1 locus were used with PCR conditions of $94^{\circ} \mathrm{C}$ for 2 min, 30 cycles of $94{ }^{\circ} \mathrm{C}$ for $30 \mathrm{~s}, 60^{\circ} \mathrm{C}$ for $60 \mathrm{~s}$, and $72{ }^{\circ} \mathrm{C}$ for $30 \mathrm{~s}$, followed by $72{ }^{\circ} \mathrm{C}$ for $7 \mathrm{~min}$.

\section{Viral vectors}

The pAAV-EF1 $\alpha:$ :DIO-ChR2(T159C)-mCherry plasmid was donated by Dr. K. Deisseroth, Stanford University. The pAAV-TRE2G::DIO-ChR2(T159C)-mCherry plasmid was constructed by replacing the human elongation factor $1 \propto(E F 1 \alpha)$ promotor sequence with the second generation tetracycline-responsive element (TRE2G) promotor sequence derived from the pLenti6PW-TGB plasmid $[12,34]$. The EF1 $\alpha$ promoter sequence was removed from the pAAV-EF1 $\alpha:$ :DIO-ChR2(T159C)-mCherry plasmid using MluI and KpnI sites. The TRE2G promotor sequence was prepared from the pLenti6PW-TGB plasmid using EcoRI and BstXI sites. Obtained TRE2G sequence and adeno-associated virus (AAV) backbone with DIOChR2(T159C)-mCherry sequences were blunted with the Klenow fragment of Escherichia coli DNA polymerase I. The TRE2G sequence was then subcloned into AAV backbone with DIO-ChR2(T159C)-mCherry sequences, generating the pAAV-TRE2G::DIO-ChR2(T159C)-m Cherry plasmid.

The recombinant AAV vectors were then produced as described previously $[35,36]$. Mice were injected with 
AAV9-TRE2G::DIO-ChR2(T159C)-mCherry at a titer of $1.3 \times 10^{13}$ viral genomes $(\mathrm{vg}) / \mathrm{ml}$ or AAV9-EF1 $\alpha::$ DIOChR2(T159C)-mCherry at a titer of $1.8 \times 10^{12}$ to $5.4 \times 10^{12} \mathrm{vg} / \mathrm{ml}$.

\section{Stereotactic surgery and cannula placement}

Surgeries were carried out as described previously $[12,13,17,37]$. The mice were anesthetized with isoflurane, given intraperitoneal injections of medetomidine hydrochloride $(0.75 \mathrm{mg} / \mathrm{kg}$ of body weight), midazolam ( $4 \mathrm{mg} / \mathrm{kg}$ of body weight), and butorphanol tartrate $(5 \mathrm{mg} /$ $\mathrm{kg}$ of body weight) and then placed in a stereotactic apparatus (Narishige, Tokyo, Japan). For the optical stimulation experiments, $0.3 \mu$ of AAV9-TRE2G::DIO-ChR2(T159C)-m Cherry was bilaterally injected into the CA3 region (anterior-posterior [AP], $-2.0 \mathrm{~mm}$; medial-lateral [ML], $\pm 2.3 \mathrm{~mm}$ from bregma; doral-ventral [DV], $-2.0 \mathrm{~mm}$ from dura) using a glass micropipette filled with mineral oil attached to a 10- $\mu$ l Hamilton microsyringe. A microsyringe pump (Narishige, Tokyo, Japan) and its controller were used to control the speed of the injection $(0.1 \mu \mathrm{l} / \mathrm{min})$. The needle was slowly lowered to the target site and remained there for $3 \mathrm{~min}$ after the injection. Then, stainless steel guide cannulas (internal diameter, $0.29 \mathrm{~mm}$; outer diameter, $0.46 \mathrm{~mm}$; Plastics One, Roanoke, VA) were bilaterally implanted in the CA3 areas (AP, $-2.0 \mathrm{~mm} ; \mathrm{ML}, \pm 2.3 \mathrm{~mm}$; DV, $-1.0 \mathrm{~mm}$ from bregma). Microscrews were anchored in the skull near bregma and lambda, and the guide cannulas were fixed in place with dental cement. After the surgery, dummy cannulas with caps were inserted into the guide cannulas as protective covers. Mice were 11-18 weeks old at the time of surgery, and were allowed to recover 16-19 weeks before being used in behavioral experiments.

To establish the labeling system (for ChR2-mCherrypositive cell counting), the same surgical procedure was used except that the cannulas were not implanted. For this, the mice were 17-27 weeks old at the time of surgery and allowed to recover $5-10$ weeks before being used in behavioral experiments.

For in vivo recording experiments, $0.3 \mu \mathrm{l}$ of AAV9-EF1 $\alpha:: D I O-C h R 2(T 159 C)$-mCherry was injected into the $\mathrm{CA} 3$ regions of the right hemispheres $(\mathrm{AP},-2.0 \mathrm{~mm}$; $\mathrm{ML},+2.3 \mathrm{~mm}$ from bregma; DV, $-2.0 \mathrm{~mm}$ from dura) using a glass micropipette. The mice were $13-18$ weeks old at the time of surgery and were allowed to recover 21-41 weeks before being used in in vivo recording experiments.

\section{Behavioral analyses and optical stimulation}

The home cage of each mouse was placed on a desk in the animal housing room for approximately $10 \mathrm{~min}$ before being transferred to the adjoining experimental room. For behavioral tests, each mouse was gently caught at the base of its tail and transferred to each context described below.
Context A was a cylindrical chamber (diameter, 180 $\mathrm{mm}$; height, $230 \mathrm{~mm}$ ) with a white acrylic floor and walls covered with black tape (see Additional file 1). Context $B$ was a square-type chamber $(175 \times 165 \mathrm{~mm}$; height, $300 \mathrm{~mm}$ ) with a transparent acrylic board front, white sides and back walls, and a floor consisting of 26 stainless steel rods with a diameter of $2 \mathrm{~mm}$ placed $5 \mathrm{~mm}$ apart with a scented tray containing $0.25 \%$ benzaldehyde underneath (Additional file 1). The rods were connected to a shock generator via a cable harness. Constant minimal illumination was provided by a small light in the chamber. Context $C$ was a square-type chamber $(290 \times$ $250 \mathrm{~mm}$; height, $290 \mathrm{~mm}$ ) with a transparent acrylic board front wall partially covered with white tape, grey side and back walls, and a floor consisting of grey acrylic board covered with white Kimtowels (Additional file 1). The room lights were off for context B but on for contexts $\mathrm{A}$ and $\mathrm{C}$.

The c-fos::tTA/KA1::Cre double transgenic mice were 11-18 weeks old and 17-27 weeks old at the time of surgery for the optical stimulation experiment and for the establishment of the labeling system (ChR2-mCherrypositive cells counting), respectively. Cannula-implanted and AAV-injected c-fos::tTA/KA1::Cre double transgenic mice were maintained on $40 \mathrm{mg} / \mathrm{kg}$ Dox food pellets in a microisolation rack system (FRP BIO2000, CLEA Japan) consisting of 16 individually ventilated boxes (1-4 cages/ box) with glass fiber filters. Mice were used for behavioral analysis 16-19 weeks and 5-10 weeks after surgery for the optical stimulation experiment and for the establishment of the labeling system (ChR2-mCherry positive cells counting), respectively. Before starting behavioral experiment, the mice were kept under the condition of Dox withdrawal (OFF Dox) for 2 days and then exposed to context A for $6 \mathrm{~min}$. One day later, the mice were subjected to contextual fear conditioning (CFC) in context $\mathrm{B}$, consisting of 3 unsignaled foot shocks (2-s duration, 0.4 $\mathrm{mA}, 1 \mathrm{~min}$ apart) beginning $2 \mathrm{~min}$ after acclimation. After the last shock, the mice remained in the context for $1 \mathrm{~min}$ and were then returned to their home cages. One day later, the mice were anesthetized with approximately $2.0 \%$ isoflurane, and the dummy cannulas were replaced with two-branch-type optical fiber units comprising a plastic cannula body and a tightly connected $0.25-\mathrm{mm}$-diameter optic fiber (COME2-DF2-250; Lucir, Ibaraki, Japan). The tip of the optical fiber was targeted slightly above CA3 (DV, $-1.5 \mathrm{~mm}$ from bregma). The mice were then returned to their home cages for $1-1.5 \mathrm{~h}$. For the optical stimulation session, the mice were moved to the experimental room, and the fiber unit was connected to an optical swivel (COME2-UFC; Lucir), which was connected to a laser ( $200 \mathrm{~mW}, 473 \mathrm{~nm}$, COME-LB473/200; Lucir) via a main optical fiber. The delivery of laser pulses was controlled by a schedule stimulator (COME2-SPG-2; Lucir) operating in 
time-lapse mode. The mice in their home cages were subjected to ten trains of laser pulses, each consisting of $300,500-\mu$ s pulses at $20-\mathrm{Hz}$ of $473 \mathrm{~nm}$ light (approximately $10 \mathrm{~mW}$ output from the fiber tip) with 45-s intertrain intervals. Approximately $1-1.5 \mathrm{~h}$ after the laser stimulation, the mice were anesthetized with approximately $2.0 \%$ isoflurane, the optic fiber unit was detached, and the mice were again returned to their home cages. The mice were then given food containing $1000 \mathrm{mg} / \mathrm{kg}$ Dox for 2 days and then maintained on food containing $40 \mathrm{mg} / \mathrm{kg}$ Dox.

To test fear memory, the mice were placed in contexts $A, B$, and $C$ for 3 min each at 1,2 , and 3 days after the optical stimulating session, respectively. At the end of each session, the mice were returned to their home cages and the contexts were cleaned with water and $80 \%$ ethanol. A video tracking system (Muromachi Kikai, Tokyo, Japan) was used to measure the freezing behavior of the animals, as described in previous studies [12, 13, 17, 38, 39]. Freezing was defined as no movement detected for $>1.5 \mathrm{~s}$. All training and testing were conducted during the light phase of the light-dark cycle. The mean values of the freezing responses during each session were analyzed except for the context A pre-exposure session, for which freezing responses during first 3 min were analyzed.

For the animals used for ChR2-mCherry-positive cell counting, the behavioral experiments were conducted as described above until CFC in context B. One day after $\mathrm{CFC}$, the mice were perfused and histologically analyzed.

\section{In vivo recordings}

In vivo recordings were carried out as described previously [17, 38-42], with modification for the optogenetic stimulation. Mice previously infected with AAV9-EF1 $\alpha:$ : DIO-ChR2(T159C)-mCherry were anesthetized with urethane $(1.2 \mathrm{~g} / \mathrm{kg})$ and placed in a stereotactic apparatus. An optic fiber (COME2-DF1-250; Lucir) was glued to the recording tungsten electrode (WE $40 \mathrm{~mm} 0030.1 \mathrm{~B} 5$, $100 \mathrm{k} \Omega$; MicroProbes, Gaithersburg, MD) so that the tip of the fiber was $500 \mu \mathrm{m}$ above the tip of the electrode. The optrode was slowly inserted into the CA3 region of the right hemisphere, and the optic fiber unit was connected to an optical swivel (COME2-UFC) connected to a laser (COME-LB473/200). The body temperatures of the mice were maintained by keeping them on a heating pad (MK-900; Muromachi Kikai, or ATC-TY; UniqueMedical Inc., Tokyo, Japan) during the recording sessions.

The delivery of laser pulses was controlled by a Master 8 device (A.M.P. Instruments, Jerusalem, Israel). Optically evoked field excitatory postsynaptic potentials (fEPSPs) was recorded by using optical test pulses (stimulation frequency, $0.033-\mathrm{Hz}, 500-\mu \mathrm{s}$ pulses). After establishing a stable baseline at the recording site for $30 \mathrm{~min}$, a $20-\mathrm{Hz}$ optical stimulating protocol was conducted, which was followed by $0.033-\mathrm{Hz}$ test pulses for $3 \mathrm{~h}$. The $20-\mathrm{Hz}$ optical stimulating protocol was identical to that used in the behavioral analysis, except the laser power was the same as that used for the optical test pulses. The stimulation laser power was adjusted to approximately half of the maximum fEPSP amplitude.

Signals were amplified and filtered from $5-\mathrm{Hz}$ to $1-\mathrm{kHz}$ with a Bioelectric amplifier (MEG-1200; Nihon Kohden, Tokyo, Japan), digitized by Digidata (1322A or 1550B; Axon Instruments, Molecular Devices, San Jose, $\mathrm{CA}$ ), and sampled at $10-\mathrm{kHz}$ using Clampex software (version 9.2 or 10.7). The data were analyzed with Clampfit 10.7 software. All animals were perfused after the recordings, and the positions of the recording sites were verified.

\section{Histology}

The mice were deeply anesthetized with an overdose of pentobarbital solution and perfused transcardially with $4 \%$ paraformaldehyde in phosphate-buffered saline (PBS; $\mathrm{pH}$ 7.4). The brains were removed and further post-fixed by immersion in $4 \%$ paraformaldehyde in PBS for $24 \mathrm{~h}$ at $4{ }^{\circ} \mathrm{C}$. Each brain was equilibrated in $25 \%$ sucrose in PBS and then frozen in dry-ice powder. Coronal sections of $30 \mu \mathrm{m}$ (for ChR2-mCherry-positive cell counting) or $50 \mu \mathrm{m}$ thickness (after in vivo recording) were cut on a cryostat and transferred to 12-well cell culture plates (Corning, Corning, NY) containing PBS. After washing with PBS, the floating sections were treated with $4^{\prime}, 6$-diamidino-2-phenylindole (DAPI) $(1 \mu \mathrm{g} / \mathrm{ml}, 10,236,276,001$; Roche Diagnostics) at room temperature for $20 \mathrm{~min}$ and then washed with PBS three times (3 min per wash). The sections were mounted on slide glass with ProLong Gold antifade reagent (Invitrogen of Thermo Fisher Scientific, Waltham, MA). Images were acquired on a fluorescence microscope (BZ9000; Keyence, Osaka, Japan) with a Plan-Apochromat $20 \times$ objective lens (Nikon, Tokyo, Japan) for ChR2-mCherry-positive cell counting or with a Plan-Apochromat $10 \times$ objective lens (Nikon) for verification of the recording site. To quantify the number of ChR2-mCherry-positive cells, images of CA3 were acquired by collecting z-stacks $(2.4 \mu \mathrm{m}$ apart, 5-6 images). Maximum intensity projections of the images were created with the image analysis software (BZ-II; Keyence). Two sections (AP, approximately -1.9 and $2.0 \mathrm{~mm}$ from bregma) corresponding to each region of interest (ROI) (CA3 pyramidal cell layers from both hemispheres within $540 \times 720 \mu \mathrm{m}^{2}$ ) were chosen from each mouse, and the ChR2-mCherry-positive cells in the ROI were counted manually. The average number of ChR2-mCherry-positive cells per section from one hemisphere are presented throughout the text.

\section{Statistical analysis}

Statistical analyses were performed using GraphPad Prism 6 (GraphPad Software, Inc., La Jolla, CA). Comparisons of 
data between two groups were analyzed with Student's $t$ tests (two tailed) or Welch's $t$ test (two tailed, without assuming homogeneity of variances), and multiple-group comparisons were assessed using one-way analyses of variance (ANOVAs) followed by Tukey's post hoc multiplecomparisons tests when significant main effects were detected. In Fig. 3e, data were analyzed with two-way repeated measures (RM) ANOVA. When significant main effects were detected, Bonferroni's multiple comparisons test were used for post hoc tests. Quantitative data are expressed as the means \pm standard errors of the means (SEMs).

\section{Results}

\section{A system for manipulation of cell ensembles in CA3}

We used c-fos::tTA/KA1::Cre double transgenic mice infected with AAV vectors harboring a light-activated cation channel, ChR2 [43], to genetically label CA3 cell ensembles corresponding to specific events (Fig. 1a). In these mice, the expression of ChR2 from the viral vector is driven by the immediate early gene c-fos, which is induced by neural activity. In the absence of Dox, tTA binds to the TRE to drive the expression of the target gene specifically in the CA3 region of the hippocampus via the Cre-loxP system from the KA1::Cre mice [29]. Therefore, the subpopulation of cells in $\mathrm{CA} 3$ activated during learning will express ChR2-mCherry (Fig. 1b, c, and d). Specifically, neural activity was elicited during context A pre-exposure and CFC in context B (referred to as "two events") when the animals were off Dox. ChR2-mCherry-positive cells were only observed in OFF Dox animals, and the number of ChR2-mCherry-positive cells were significantly increased by the two events (Fig. $1 \mathrm{~d}$ and e; 4 mice/ group, one-way ANOVA; $F_{2,9}=13.4, p=0.0020$; Tukey's post hoc multiple-comparisons test, OFF Dox, two events vs. ON Dox, two events, $p=0.0016$ ). For the OFF Dox condition, the labeling efficiency in mice kept in their home cages was significantly lower than in those exposed to the two events (Tukey's post hoc multiple-comparisons test, OFF Dox, two events vs. OFF Dox, home cage, $p=0.0256$ ).
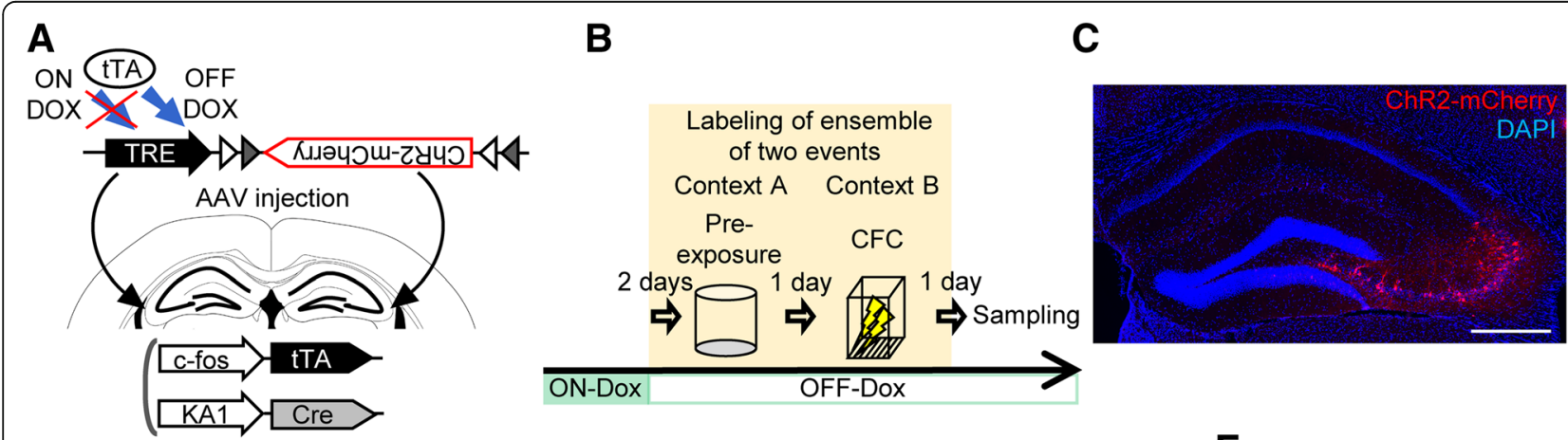

D

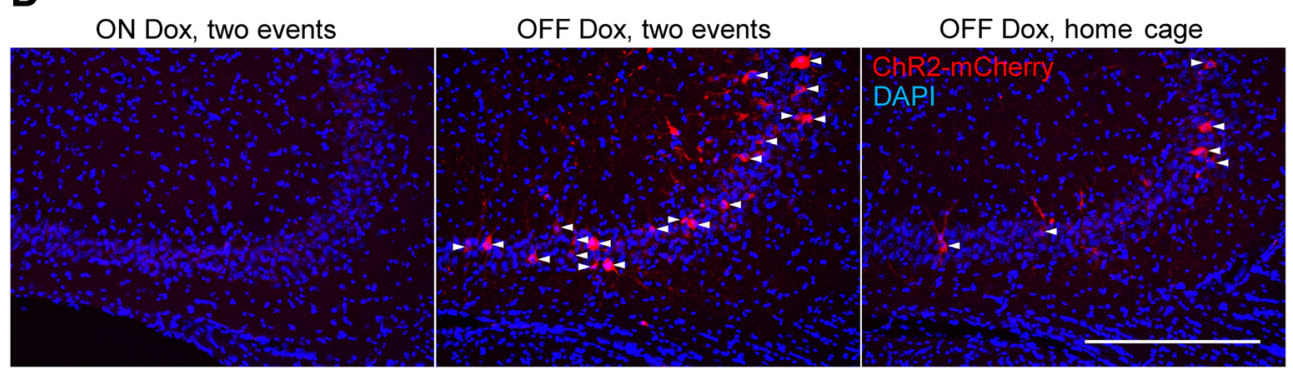

E

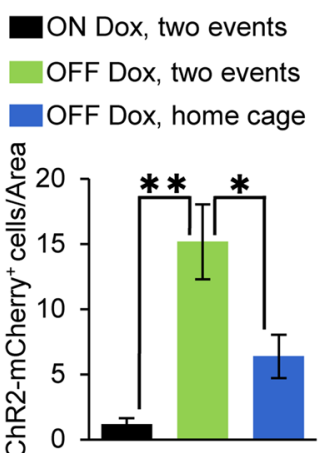

Fig. 1 System for activity-dependent labeling of CA3 neurons. a Diagram showing the activity-dependent labeling of neurons in c-fos:: tTA/ KA1::Cre double transgenic mice with an AAV system. Gray and white triangles represent loxP and lox2272 sequences, respectively. $\mathbf{b}$ Scheme of the experiment. Neural activity was elicited during context A pre-exposure and CFC in context B (i.e., the two events) in animals off of doxycycline treatment (OFF Dox). c Representative image of ChR2-mCherry expression in the CA3 region of the hippocampus of an AAV-injected c-fos::tTA/KA1::Cre double transgenic mouse exposed to the two events in the OFF Dox condition. Scale bar, $500 \mu \mathrm{m}$. $\mathbf{d}$ Representative images of ChR2-mCherry expression in the CA3 regions of AAV-injected c-fos::TTA/KA1::Cre double transgenic mice exposed to the two events in an ON Dox condition (left) or an OFF Dox condition (middle) and in mice in the home cage in the OFF Dox condition (right). The ChR2-mCherry signal is in red and DAPI nuclear staining is in blue. Arrowheads indicate ChR2-mCherry-positive cells. Scale bar, $300 \mu \mathrm{m}$. e Graph showing the average numbers of ChR2-mCherry-positive cells per section in the CA3 region from one hemisphere (counted from both hemisphere, two sections from 4 mice/group, one-way ANOVA; $F_{2,9}=13.4, p=0.0020 ;$ Tukey's post hoc multiple-comparisons test, ${ }^{*} p<0.05,{ }^{* *} p<0.01$ ) 
These data show that the cell ensembles in CA3 corresponding to the two events are specifically labeled with this system.

\section{Synchronous activation of cell ensembles in CA3 associates distinct events}

The CA3-specific labeling system was used to examine whether the simultaneous activation of cell ensembles in this region corresponding to each of the two events (i.e., the context A pre-exposure and CFC in context B) generates an artificial association between them. For this, the c-fos::tTA/KA1::Cre double transgenic mice harboring the ChR2-mCherry viral vector and guide cannulas in bilateral CA3 regions (Fig. 2a) were divided into two groups, a laser ON group $(n=12)$ subjected to optical stimulation and a laser OFF group $(n=10)$, which received no laser stimulation as a control. After AAV injections and cannula implantations, the mice were taken off Dox to enable neural activity-dependent labeling of cells and then subjected to the two events as described above (Fig. 2b). During these sessions, there were no significant differences in freezing between the laser $\mathrm{ON}$ and laser OFF groups (Fig. 2c-e; context A pre-exposure session, unpaired Student's $t$ test; $t_{20}=1.255, p=0.2239$; pre-foot shock session during CFC, unpaired Student's $t$ test; $t_{20}=0.6455, p=0.5259$; during and after foot shock session of CFC, Welch's $t$ test; $t_{12.62}=0.2795, p=$ 0.7844). One day after CFC, the laser ON group was subjected to bilateral $20-\mathrm{Hz}$ laser stimulation of CA3 in their home cages to synchronously activate all cell ensembles. Mice in the laser OFF group were attached to optic fibers targeting CA3, but the laser pulses were not delivered.

Beginning the day after the optical stimulation, the mice were subjected to fear memory tests over three consecutive days using the different contexts. When mice were tested in context A, in which they had not experienced CFC, the laser ON group showed significantly

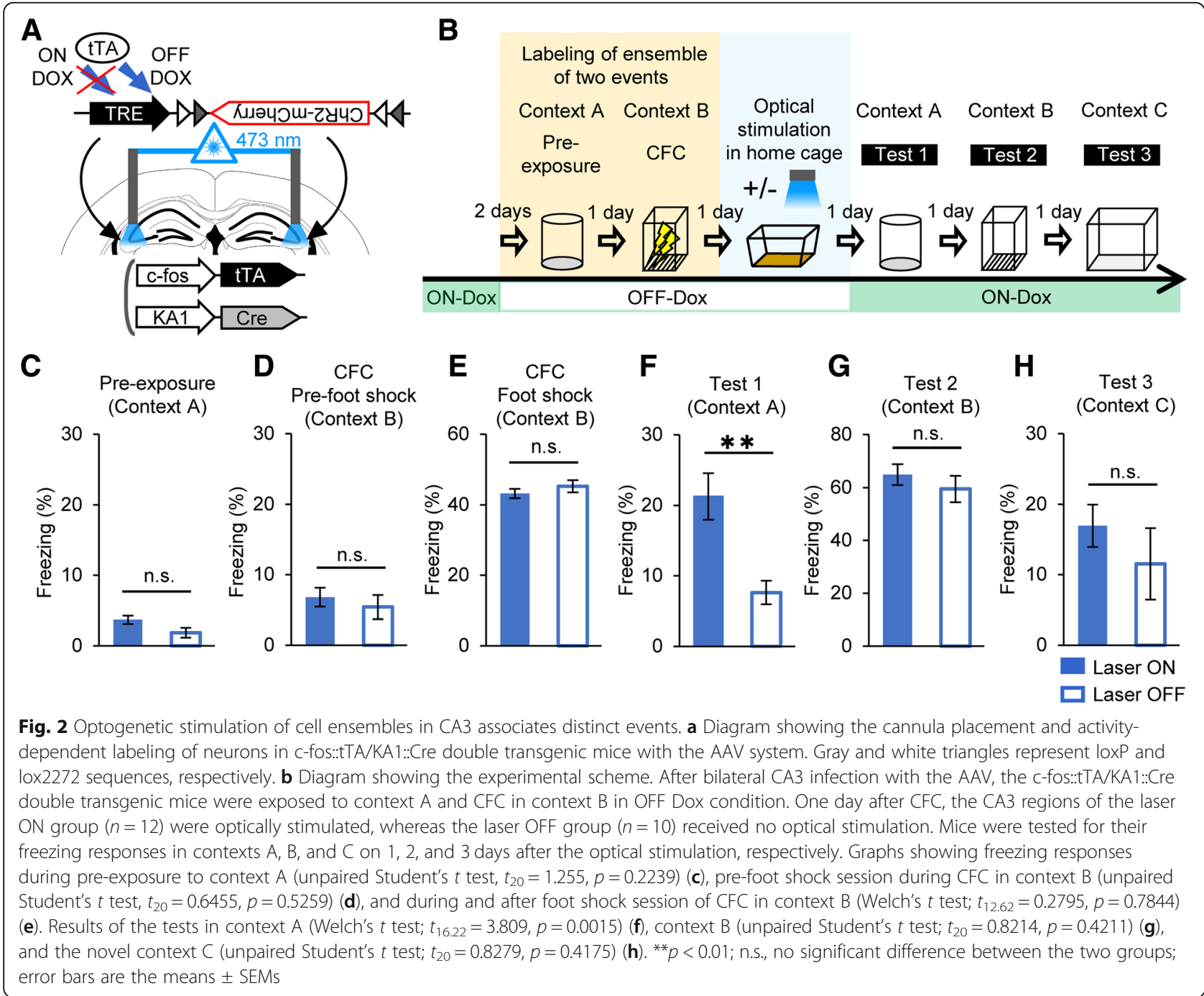


more freezing than the laser OFF group (Fig. 2f; Welch's $t$ test, $t_{16.22}=3.809, p=0.0015$ ). In context $\mathrm{B}$, in which they were subjected to CFC, there was no difference in freezing between the two groups (Fig. 2g; unpaired Student's $t$ test; $t_{20}=0.8214, p=0.4211$ ). Furthermore, there was no significant difference in freezing in the novel context C (Fig. 2h, unpaired Student's $t$ test; $t_{20}=0.8279$, $p=0.4175)$, demonstrating that the observed fear memory was context specific. These results indicate that two distinct events can be associated via the synchronous activation of the corresponding cell ensembles in CA3.

\section{Optogenetic stimulation induces LTP within the CA3-CA3 recurrent circuit}

To study whether the $20-\mathrm{Hz}$ optogenetic stimulation that induced the artificial association described above also induces LTP at CA3-CA3 synapses, in vivo extracellular recordings were performed in the $\mathrm{CA} 3$ regions of anesthetized KA1::Cre mice or CA3-NR1 KO mice [29] infected AAV-EF1 $\alpha:$ DIO-ChR2-mCherry in CA3 (Fig. 3a, b). It is reported that LTP at CA3-CA3 recurrent synapses depends on NMDA receptor of the CA3 whereas dysfunction of NMDA receptors in the CA3 has no effect on the LTP between mossy fiber-CA3 synapses or Shaffer collateral-CA1 synapses [29, 44-46]. Therefore, CA3-NR1 $\mathrm{KO}$ mice was used to verify that the observed LTP in KA1::Cre mice is attributed to LTP within CA3-CA3 synapses.

ChR2-mCherry expression was observed in the CA3 regions of AAV-infected animals (Fig. 3c); the loci of recording electrodes were identified histologically after completion of the recordings (Additional file 2). During $20-\mathrm{Hz}$ laser stimulation, field responses followed stimulations faithfully in both the KA1::Cre mice and the CA3-NR1 KO mice (Additional file 3). There was no statistical difference of input-output function between the groups (Additional file 4; 50\% stimulation intensity, unpaired Student's $t$ test; $t_{6}=0.6807, p=0.5214 ; 100 \%$ stimulation intensity, unpaired Student's $t$ test; $t_{6}=0.8144$, $p=0.4465 ; 50 \%$ fEPSP amplitude, unpaired Student's $t$ test; $t_{6}=0.6289, p=0.5526 ; 100 \%$ fEPSP amplitude, $t_{6}=$ $0.7006, p=0.5098$ ).

A progressive increase in the fEPSP slopes was recorded in KA1::Cre mice (Fig. 3d-f; $n=4$ mice/group, -20 to -1 min vs. $40-59 \mathrm{~min}$, paired Student's $t$ test; $t_{3}=3.440, p=$ 0.0412 ; -20 to -1 min vs. 160 to 179 min, paired Student's $t$ test; $t_{3}=4.327, p=0.0228$ ) but not in CA3-NR1 KO mice (Fig. $3 \mathrm{~d}-\mathrm{f}$; -20 to $-1 \mathrm{~min}$ vs. $40-59 \mathrm{~min}$, paired Student's $t$ test; $t_{3}=0.3868, p=0.7247 ;-20$ to $-1 \mathrm{~min}$ vs. 160 to 179 min, paired Student's $t$ test; $t_{3}=0.4318, p=0.6951$ ). The fEPSP slope recorded in KA1::Cre mice was significantly higher than in CA3-NR1 KO mice after $20-\mathrm{Hz}$ laser stimulation (Fig. 3e; Two-way RM ANOVA; interaction, $\mathrm{F}_{42,129}=1.418, p=0.0709 ;$ time, $\mathrm{F}_{42,129}=0.6796, p=0.9248$, genotype; $\left.F_{1,129}=160.7, p<0.0001\right)$. This difference was found $160-175 \mathrm{~min}$ after the $20-\mathrm{Hz}$ laser stimulation (Fig. 3e, f; Bonferroni's multiple comparisons test, KA1::Cre vs. CA3-NR1 KO, $p<0.05,155-175$ min after the $20-\mathrm{Hz}$ laser stimulation; 160-179 min, unpaired Student's $t$ test; $t_{6}=2.690, p=0.036$ ). These results indicate that 20-Hz laser stimulation of ChR2-expressing neurons of the CA3 induces LTP within CA3-CA3 synapses.

\section{Discussion}

Pattern completion, a retrieval process of associative memory, is a well-known function of the recurrent network in CA3 [27-29]. Moreover, the mechanism avoiding interference between pre-stored information within CA3-CA3 recurrent synapses and new information have been advocated [47, 48]. Previous gain-of-function studies using an optogenetic technique showed that manipulation of the DG [4, 8, 21-24] or CA1 [12] engrams is important for memory reactivation and to generate synthetic or false memory by linking between stored information and sensory input by artificial activation of cell ensembles. However, CA3 gain-of-function study has not been reported and it was not known whether CA3, which has recurrent circuit is involved in the generation of associations among the stored information. Here, we show that simultaneous optogenetic activation of CA3 cell ensembles bridges two initially independent events.

Ohkawa et al. [12] induced association between prestored information within CA1 and basolateral amygdala, whereas our study induced association between pre-stored information within one brain region, CA3. Our results imply that CA3 composing recurrent circuit has unique characteristics to bridge the memories and to generate new memories among the multiple memories. To the best of our knowledge, this study is the first gain-of-function study in CA3 demonstrating that the association between individual units of stored information occurs within a single brain region, namely, the CA3 of the hippocampus.

LTP within CA3-CA3 recurrent synapses depends on NMDA receptor of CA3 whereas dysfunction of NMDA receptors in the CA3 has no effect on the LTP between mossy fiber-CA3 synapses or Shaffer collateral-CA1 synapses $[29,44-46]$. Our study showed that, $20-\mathrm{Hz}$ optogenetic stimulation induced LTP within the CA3 whereas this LTP was abolished in CA3-NR1 KO mice (Fig. 3d-f). These data indicate that, our system detects the LTP within CA3-CA3 recurrent synapses.

The subpopulation of CA3 neurons activated during contextual learning are reactivated specifically during exposure to the learned context and during memory retrieval [2]. Thus, distinct CA3 cell ensembles may be activated in distinct contexts, such as during the two events (pre-exposure and CFC) in this study. These two initially separate "memories" can be associated to generate 


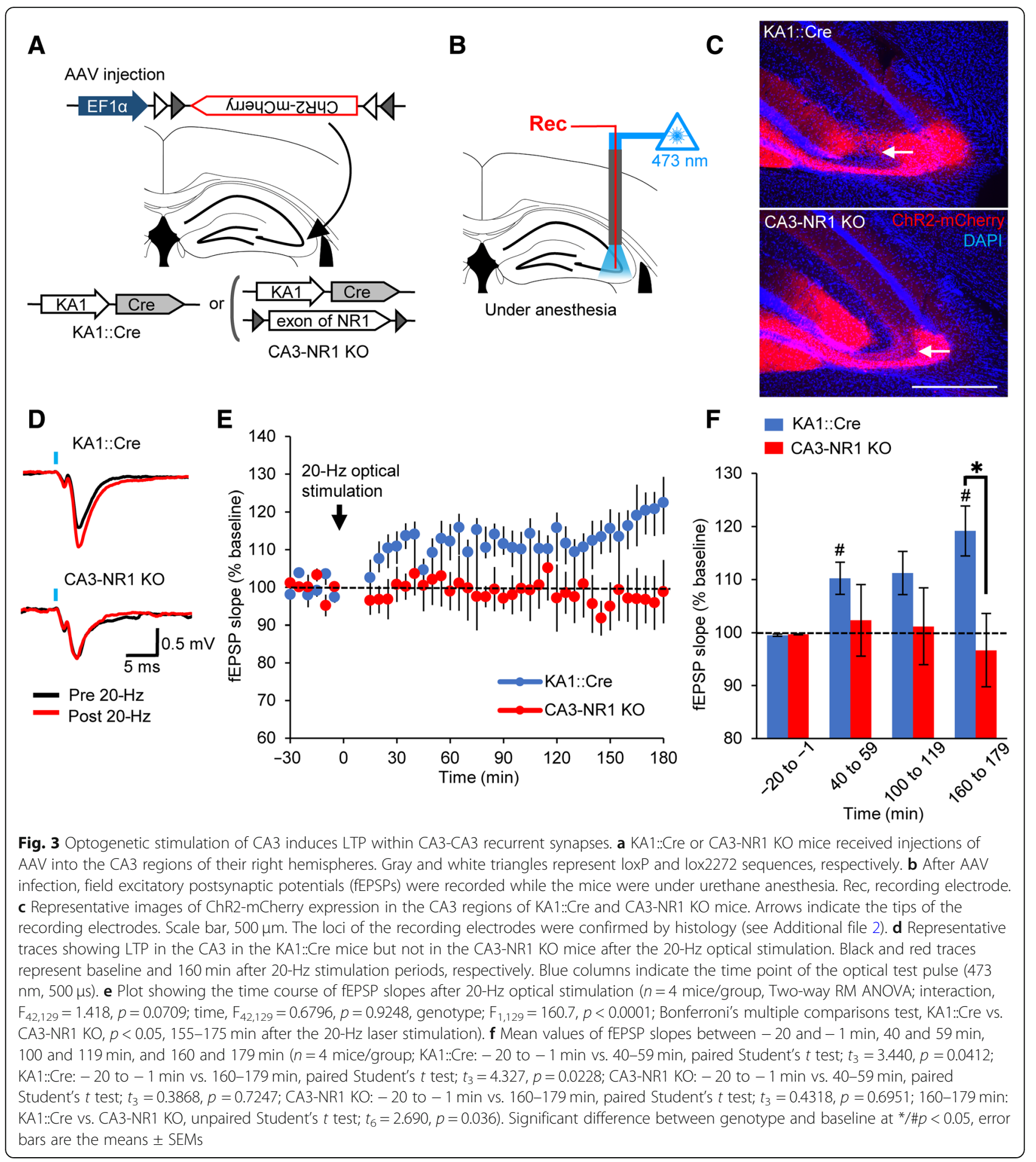

new memories by synchronous activation of the corresponding cell ensembles. The results of our electrophysiology showed that optogenetic stimulation of CA3 induce LTP within CA3 recurrent synapses. It is reported that, LTP at appropriate synapses are both necessary and sufficient for information storage $[16,17]$. Moreover, LTP also contributes to associative learning or memory update
[18-20]. A potential mechanism underlying memory association observed in our study is that optogenetic stimulation increased synaptic efficacy between cell ensembles within the CA3 recurrent circuit, generating new functional connection. This newly generated connection leads to the activation of the cell ensemble corresponding to the pre-exposure followed by the activation of 
the cell ensemble corresponding to CFC. Thus, recall of the pre-exposure memory may triggers the recall of the CFC memory and induces freezing behavior. The sharing of a memory ensemble may also emerge in regions downstream of CA3 to associate the events [12, 13, 37, 49, 50].

The results of this study show that stimulation at $20-\mathrm{Hz}$, a frequency lower than that used for theta burst stimulation, is sufficient to induce LTP in the CA3. A recent study reported that CA3-CA3 synapses exhibit a unique "symmetrical" spike timing-dependent plasticity curve, in which LTP is induced regardless of pre-post spike timing with a relatively large interval (half-width, $\sim 150 \mathrm{~ms}$ ) [28]. This spike timing-dependent plasticity may have contribute to the induction of LTP observed here with $20-\mathrm{Hz}$ (50-ms interval) optogenetic stimulation. Further study is needed to determine the contribution of spike timing-dependent plasticity in the CA3 recurrent circuit to associative memory.

\section{Additional files}

Additional file 1: Contexts used for the behavioral experiments. Photographs showing contexts A, B, and C used in this study. (PDF 2587 kb)

Additional file 2: Histological verification of electrode tips for in vivo electrophysiological recording. Placements of recording electrode tips (red circles) in KA1::Cre (left) and CA3-NR1 KO (right) mice. (PDF 3849 kb)

Additional file 3: In vivo optically evoked synaptic responses in CA3. Envelopes of field responses to $20-\mathrm{Hz}$ optical stimulation, obtained from KA1::Cre (top) and CA3-NR1 KO (bottom) animals infected with AAVEF1a::DIO-ChR2-mCherry in the CA3 region. Note that the responses follow the stimulation faithfully. Blue columns indicate the timing of laser stimulation. (PDF $1486 \mathrm{~kb}$ )

Additional file 4: Input-output function. Data of input-output function obtained from KA1::Cre and CA3-NR1 KO mice infected with AAV-EF1a::DIOChR2-mCherry in the CA3 region. Laser power and fEPSP amplitude at the time of getting maximum fEPSP amplitude and at the time of using 50\% of this stimulation intensity are shown. There was no statistical difference of input-output function between the groups (50\% stimulation intensity, unpaired Student's $t$ test; $t_{6}=0.6807, p=0.5214 ; 100 \%$ stimulation intensity, unpaired Student's $t$ test; $t_{6}=0.8144, p=0.4465 ; 50 \%$ fEPSP amplitude, unpaired Student's $t$ test; $t_{6}=0.6289, p=0.5526 ; 100 \%$ fEPSP amplitude, $\left.t_{6}=0.7006, p=0.5098\right)$. (XLS $\left.27 \mathrm{~kb}\right)$

\section{Funding}

This work was supported by a grant-in-aid for Scientific Research on Innovative Areas ("Memory dynamism," JP25115002 to K.I.) from the Ministry of Education, Culture, Sports, Science, and Technology of Japan (MEXT), the Japan Society for the Promotion of Science (JSPS; KAKENHI grant numbers JP23220009 and JP18H05213 to K.I. and JP16H04653 to N. Ohkawa), the Core Research for Evolutional Science and Technology (CREST) program of the Japan Science and Technology Agency (JST; JPMJCR13W1 to K.I.), the Precursory Research for Embryonic Science and Technology (PRESTO) program of JST (JPMJPR1684 to N. Ohkawa), the JSPS KAKENHI Challenging Research exploratory grant (JP17K19445 to M.N.), the Mitsubishi Foundation (support to K.I.), the Uehara Memorial Foundation (support to K.I.), the Takeda Science Foundation (support to K.I. and M.N.), The Hokuriku Bank grant-in-aid for Young Scientists (to M.N.), the Tamura Science and Technology Foundation (support to M.N.), and the Narishige Neuroscience Research Foundation (support to M.N.)

Availability of data and materials

The datasets used and/or analyzed during the current study are available from the corresponding author on reasonable request.

\section{Authors' contributions}

N. Oishi, M.N., N. Ohkawa, and K.I. designed the study. N. Oishi conducted surgeries, behavioral experiments, histological experiments, in vivo recordings and analyses of all the data. N. Oishi, M.N., Y. Saitoh, and N. Ohkawa designed the in vivo recording experiments. Y. Sano constructed the plasmids. S.T., H.N., and M.M. contributed to the production and maintenance of the transgenic animals. S.M. prepared the AAV vectors. N. Oishi, M.N., and K.I. wrote the manuscript. K.I. supervised the entire project. All authors read and approved the final manuscript.

\section{Ethics approval}

All procedures involving the use of animals complied with the guidelines of the National Institutes of Health and were approved by the Animal Care and Use Committee of the University of Toyama.

Consent for publication

Not applicable.

\section{Competing interests}

S.M. owns equity in a company (Gene Therapy Research Institution) that commercializes the use of AAV vectors for gene therapy applications. To the extent that the work in this manuscript increases the value of these commercial holdings, S.M. has a conflict of interest. The other authors declare no competing interests.

\section{Publisher's Note}

Springer Nature remains neutral with regard to jurisdictional claims in published maps and institutional affiliations.

\section{Author details}

'Department of Biochemistry, Graduate School of Medicine and Pharmaceutical Sciences, University of Toyama, Toyama 930-0194, Japan. ${ }^{2}$ Core Research for Evolutional Science and Technology (CREST), Japan Science and Technology Agency (JST), University of Toyama, Toyama 930-0194, Japan. ${ }^{3}$ Precursory Research for Embryonic Science and Technology (PRESTO), JST, University of Toyama, Toyama 930-0194, Japan. ${ }^{4}$ Division of Animal Experimental Laboratory, Life Science Research Center, University of Toyama, Toyama 930-0194, Japan. ${ }^{5}$ Division of Neurology, Department of Medicine, Jichi Medical University, Tochigi 329-0498, Japan. ${ }^{6}$ Center for Gene and Cell Therapy, The Institute of Medical Science, The University of Tokyo, Tokyo 108-8639, Japan. ${ }^{7}$ Present address: Department of Applied Biological Science, Faculty of Science and Technology, Tokyo University of Science, Noda, Chiba 278-8510, Japan.

\begin{abstract}
Acknowledgements
We thank S. Tonegawa (RIKEN-Massachusetts Institute of Technology) and Itohara (RIKEN Brain Science Institute) for the floxed-NR1 mice, K. Deisseroth Stanford University) for the ChR2(T159C) AAV vector, H. Hioki and T. Kanek (Kyoto University) for the TRE-EGFP LV vector, M. Ito and N. Takino (Jichi Medica University) for their help with production of the AAV vectors, K. Koga (Hyogo College of Medicine) for support for electrophysiological experiment. We thank all members of the Inokuchi laboratory for daily discussions and advice.
\end{abstract}


Received: 25 October 2018 Accepted: 26 December 2018 Published online: 08 January 2019

\section{References}

1. Deng W, Mayford M, Gage FH. Selection of distinct populations of dentate granule cells in response to inputs as a mechanism for pattern separation in mice. Elife. 2013;2:e00312.

2. Denny CA, Kheirbek MA, Alba EL, Tanaka KF, Brachman RA, Laughman KB, Tomm NK, Turi GF, Losonczy A, Hen R. Hippocampal memory traces are differentially modulated by experience, time, and adult neurogenesis. Neuron. 2014:83(1):189-201.

3. Guzowski JF, McNaughton BL, Barnes CA, Worley PF. Environment-specific expression of the immediate-early gene arc in hippocampal neuronal ensembles. Nat Neurosci. 1999;2(12):1120-4.

4. Ramirez S, Liu X, Lin PA, Suh J, Pignatelli M, Redondo RL, Ryan TJ, Tonegawa S. Creating a false memory in the hippocampus. Science. 2013;341(6144): 387-91.

5. Reijmers LG, Perkins BL, Matsuo N, Mayford M. Localization of a stable neural correlate of associative memory. Science. 2007;317(5842):1230-3.

6. Tayler KK, Tanaka KZ, Reijmers LG, Wiltgen BJ. Reactivation of neural ensembles during the retrieval of recent and remote memory. Curr Biol. 2013;23(2):99-106.

7. Han JH, Kushner SA, Yiu AP, Hsiang HL, Buch T, Waisman A, Bontempi B, Neve RL, Frankland PW, Josselyn SA. Selective erasure of a fear memory. Science. 2009;323(5920):1492-6.

8. Liu X, Ramirez S, Pang PT, Puryear CB, Govindarajan A, Deisseroth K, Tonegawa S. Optogenetic stimulation of a hippocampal engram activates fear memory recall. Nature. 2012;484(7394):381-5.

9. Rogerson T, Cai DJ, Frank A, Sano Y, Shobe J, Lopez-Aranda MF, Silva AJ. Synaptic tagging during memory allocation. Nat Rev Neurosci. 2014;15(3): 157-69.

10. Silva AJ, Zhou Y, Rogerson T, Shobe J, Balaji J. Molecular and cellular approaches to memory allocation in neural circuits. Science. 2009; 326(5951):391-5.

11. Garner AR, Rowland DC, Hwang SY, Baumgaertel K, Roth BL, Kentros C, Mayford M. Generation of a synthetic memory trace. Science. 2012; 335(6075):1513-6.

12. Ohkawa N, Saitoh $Y$, Suzuki A, Tsujimura S, Murayama E, Kosugi S, Nishizono H, Matsuo M, Takahashi Y, Nagase M, et al. Artificial association of pre-stored information to generate a qualitatively new memory. Cell Rep. 2015;11(2): 261-9.

13. Yokose J, Okubo-Suzuki R, Nomoto M, Ohkawa N, Nishizono H, Suzuki A, Matsuo M, Tsujimura S, Takahashi Y, Nagase M, et al. Overlapping memory trace indispensable for linking, but not recalling, individual memories. Science. 2017:355(6323):398-403.

14. Caporale N, Dan Y. Spike timing-dependent plasticity: a Hebbian learning rule. Annu Rev Neurosci. 2008:31:25-46.

15. Hebb DO. The organization of behavior; a neuropsychological theory. New York: Wiley; 1949.

16. Nabavi S, Fox R, Proulx CD, Lin JY, Tsien RY, Malinow R. Engineering a memory with LTD and LTP. Nature. 2014;511(7509):348-52.

17. Abdou K, Shehata M, Choko K, Nishizono H, Matsuo M, Muramatsu SI, Inokuchi K. Synapse-specific representation of the identity of overlapping memory engrams. Science. 2018;360(6394):1227-31.

18. Whitlock JR, Heynen AJ, Shuler MG, Bear MF. Learning induces long-term potentiation in the hippocampus. Science. 2006:313(5790):1093-7.

19. Broussard Jl, Yang K, Levine AT, Tsetsenis T, Jenson D, Cao F, Garcia I, Arenkiel BR, Zhou FM, De Biasi M, et al. Dopamine regulates aversive contextual learning and associated in vivo synaptic plasticity in the Hippocampus. Cell Rep. 2016;14(8):1930-9.

20. Nader K, Hardt O. A single standard for memory: the case for reconsolidation. Nat Rev Neurosci. 2009;10(3):224-34.

21. Redondo RL, Kim J, Arons AL, Ramirez S, Liu X, Tonegawa S. Bidirectional switch of the valence associated with a hippocampal contextual memory engram. Nature. 2014;513(7518):426-30.

22. Roy DS, Arons A, Mitchell TI, Pignatelli M, Ryan TJ, Tonegawa S. Memory retrieval by activating engram cells in mouse models of early Alzheimer's disease. Nature. 2016;531(7595):508-12.

23. Ryan TJ, Roy DS, Pignatelli M, Arons A, Tonegawa S. Memory. Engram cells retain memory under retrograde amnesia. Science. 2015;348(6238):1007-13.
24. Ramirez S, Liu X, MacDonald CJ, Moffa A, Zhou J, Redondo RL, Tonegawa S. Activating positive memory engrams suppresses depression-like behaviour. Nature. 2015;522(7556):335-9.

25. Ishizuka N, Weber J, Amaral DG. Organization of intrahippocampal projections originating from CA3 pyramidal cells in the rat. J Comp Neurol. 1990;295(4):580-623.

26. Li XG, Somogyi P, Ylinen A, Buzsaki G. The hippocampal CA3 network: an in vivo intracellular labeling study. J Comp Neurol. 1994;339(2):181-208.

27. Guzman SJ, Schlogl A, Frotscher M, Jonas P. Synaptic mechanisms of pattern completion in the hippocampal CA3 network. Science. 2016;353(6304):1117-23.

28. Mishra RK, Kim S, Guzman SJ, Jonas P. Symmetric spike timing-dependent plasticity at CA3-CA3 synapses optimizes storage and recall in autoassociative networks. Nat Commun. 2016;7:11552.

29. Nakazawa K, Quirk MC, Chitwood RA, Watanabe M, Yeckel MF, Sun LD, Kato A, Carr CA, Johnston D, Wilson MA, et al. Requirement for hippocampal CA3 NMDA receptors in associative memory recall. Science. 2002;297(5579):211-8.

30. Levy WB. A sequence predicting CA3 is a flexible associator that learns and uses context to solve hippocampal-like tasks. Hippocampus. 1996;6(6):579-90.

31. Rajji T, Chapman D, Eichenbaum H, Greene R. The role of CA3 hippocampal NMDA receptors in paired associate learning. J Neurosci. 2006;26(3):908-15.

32. The Jackson Laboratory: Mouse Strain Datasheet - 006474 https://www.jax. org/strain/006474 Accessed 25 Dec 2018.

33. Matsuo N, Reijmers L, Mayford M. Spine-type-specific recruitment of newly synthesized AMPA receptors with learning. Science. 2008;319(5866):1104-7.

34. Hioki H, Kuramoto E, Konno M, Kameda H, Takahashi Y, Nakano T, Nakamura KC, Kaneko T. High-level transgene expression in neurons by lentivirus with Tet-off system. Neurosci Res. 2009;63(2):149-54.

35. lida A, Takino N, Miyauchi H, Shimazaki K, Muramatsu S. Systemic delivery of tyrosine-mutant AAV vectors results in robust transduction of neurons in adult mice. Biomed Res Int. 2013;2013:974819.

36. Li XG, Okada T, Kodera M, Nara Y, Takino N, Muramatsu C, Ikeguchi K, Urano F, Ichinose $H$, Metzger D, et al. Viral-mediated temporally controlled dopamine production in a rat model of Parkinson disease. Mol Ther. 2006;13(1):160-6.

37. Nomoto M, Ohkawa N, Nishizono H, Yokose J, Suzuki A, Matsuo M, Tsujimura S, Takahashi Y, Nagase M, Watabe AM, et al. Cellular tagging as a neural network mechanism for behavioural tagging. Nat Commun. 2016;7:12319.

38. Alam MJ, Kitamura T, Saitoh Y, Ohkawa N, Kondo T, Inokuchi K. Adult neurogenesis conserves hippocampal memory capacity. J Neurosci. 2018;38(31):6854-63.

39. Kitamura T, Saitoh Y, Takashima N, Murayama A, Niibori Y, Ageta H, Sekiguchi M, Sugiyama H, Inokuchi K. Adult neurogenesis modulates the hippocampusdependent period of associative fear memory. Cell. 2009;139(4):814-27.

40. Fukazawa Y, Saitoh Y, Ozawa F, Ohta Y, Mizuno K, Inokuchi K. Hippocampal LTP is accompanied by enhanced F-actin content within the dendritic spine that is essential for late LTP maintenance in vivo. Neuron. 2003;38(3):447-60.

41. Okubo-Suzuki R, Saitoh $Y$, Shehata M, Zhao Q, Enomoto H, Inokuchi $K$ Frequency-specific stimulations induce reconsolidation of long-term potentiation in freely moving rats. Mol Brain. 2016:9:36.

42. Shehata M, Abdou K, Choko K, Matsuo M, Nishizono H, Inokuchi K. Autophagy enhances memory erasure through synaptic destabilization. J. Neurosci. 2018;38(15):3809-22.

43. Fenno L, Yizhar O, Deisseroth K. The development and application of optogenetics. Annu Rev Neurosci. 2011;34:389-412.

44. Harris EW, Cotman CW. Long-term potentiation of Guinea pig mossy fiber responses is not blocked by $\mathrm{N}$-methyl D-aspartate antagonists. Neurosci Lett. 1986;70(1):132-7.

45. Williams S, Johnston D. Muscarinic depression of long-term potentiation in CA3 hippocampal neurons. Science. 1988;242(4875):84-7.

46. Zalutsky RA, Nicoll RA. Comparison of two forms of long-term potentiation in single hippocampal neurons. Science. 1990;248(4963):1619-24.

47. Kunec S, Hasselmo ME, Kopell N. Encoding and retrieval in the CA3 region of the hippocampus: a model of theta-phase separation. J Neurophysiol. 2005;94(1):70-82.

48. Villarreal DM, Gross AL, Derrick BE. Modulation of CA3 afferent inputs by novelty and theta rhythm. J Neurosci. 2007;27(49):13457-67.

49. Cai DJ, Aharoni D, Shuman T, Shobe J, Biane J, Song W, Wei B, Veshkini M La-Vu M, Lou J, et al. A shared neural ensemble links distinct contextual memories encoded close in time. Nature. 2016;534(7605):115-8.

50. Rashid AJ, Yan C, Mercaldo V, Hsiang HL, Park S, Cole CJ, De Cristofaro A, Yu J, Ramakrishnan C, Lee SY, et al. Competition between engrams influences fear memory formation and recall. Science. 2016;353(6297):383-7. 